\begin{tabular}{|c|c|}
\hline \multirow[t]{11}{*}{ Citation } & Marko Simicic 1,3 , Sebastien Morrison 2,3, Bertrand Parvais ${ }^{3}$, Pieter Weckx³, \\
\hline & Ben Kaczer ${ }^{3}$, Ken Sawada ${ }^{4}$, Hiroaki Ammo ${ }^{4}$, Shinya Yamakawa ${ }^{4}$, Kazuki \\
\hline & Nomoto $^{5}$, Makoto Ohno ${ }^{5}$, Dimitri Linten ${ }^{3}$, Diederik Verkest ${ }^{3}$, Piet Wambacq ${ }^{2,3}$, \\
\hline & Guido Groeseneken ${ }^{1,3}$, Georges Gielen ${ }^{1},(2017)$ \\
\hline & ${ }^{1}$ Department of Electrical Engineering (ESAT), KU Leuven, Leuven, Belgium \\
\hline & ${ }^{2}$ Vrije Universiteit Brussel, Belgium \\
\hline & ${ }^{3}$ imec, Leuven, Belgium \\
\hline & ${ }^{4}$ Sony Corporation, 4-14-1 Asahi-cho, Atsugi-shi, Kanagawa, 243-0014 Japan \\
\hline & ${ }^{5}$ Sony Corporation to IMEC, Kapeldreef 75, B-3001, Leuven, Belgium \\
\hline & A fully-integrated method for RTN parameter extraction \\
\hline & Symposium on VLSI Technology, 2017, T132--T133. \\
\hline Archived version & $\begin{array}{l}\text { Author manuscript: the content is identical to the content of the published } \\
\text { paper, but without the final typesetting by the publisher }\end{array}$ \\
\hline Published version & http://ieeexplore.ieee.org/document/7998151/ \\
\hline Journal homepage & http://vlsisymposium.org/2017/ \\
\hline Author contact & your email marko.simicic@esat.kuleuven.be \\
\hline & your phone number +32 16325854 \\
\hline
\end{tabular}




\title{
A fully-integrated method for RTN parameter extraction
}

\author{
M. Simicic ${ }^{1,3}$, S. Morrison ${ }^{2,3}$, B. Parvais 3 , P. Weckx ${ }^{3}$, B. Kaczer ${ }^{3}$, K. Sawada ${ }^{4}$, H. Ammo ${ }^{4}$, S. Yamakawa ${ }^{4}$, \\ K. Nomoto ${ }^{5}$, M. Ohno ${ }^{5}$, D. Linten ${ }^{3}$, D. Verkest ${ }^{3}$, P. Wambacq ${ }^{2,3}$, G. Groeseneken ${ }^{1,3}$, G. Gielen ${ }^{1}$ \\ ${ }^{1}$ Department of Electrical Engineering (ESAT), KU Leuven, Belgium, ${ }^{2}$ Vrije Universiteit Brussel (VUB), Dept. of Electronics and \\ Informatics (ETRO), Brussels, Belgium, ${ }^{3}$ imec, Leuven, Belgium, ${ }^{4}$ Sony Semiconductor Solutions, Kanagawa, Japan, \\ ${ }^{5}$ Sony Semiconductor Solutions to imec, Leuven, Belgium
}

\begin{abstract}
A method for on-chip extraction of random telegraph noise (RTN) parameters from transistors is proposed. Exploiting the nature of exponential distributed RTN events, the proposed circuit enables the automatic extraction of mean RTN time constants from a large array of small-area transistors. The on-chip data processing provides a simplified measurement infrastructure, reduces the measurement time by parallelization and increased efficiency, reduces the data post-processing effort and extends the measurement frequency band. The methodology is demonstrated in a prototype chip fabricated in a 28nm High-k Metal Gate (HK/MG) CMOS technology. The 1.17 $\mathrm{mm}^{2}$ chip includes two arrays of 18,144 transistors each, analog circuitry for sensing and digitizing the RTN signals and a digital signal processing block. The experimental results agree with expectations.
\end{abstract}

Keywords - Random telegraph noise, RTN, characterization

Introduction: Since RTN in scaled MOSFETs poses a serious concern for designing circuits such as SRAM, imagers and critical logic data paths [1-3], its accurate characterization is of utmost importance. RTN-induced threshold voltage shift $(\Delta V$ th $)$ can be described by a stochastic number of active traps in the gate oxide, each with a stochastic impact on the $\Delta \mathrm{V}_{\text {th }}$ [4]. As the number of traps has been shown to be Poisson-distributed with the mean $\mathrm{N}_{\mathrm{T}}$ and the single defect impact to be exponentially distributed with mean $\eta[5]$, the electrostatics of RTN, at a given bias, can be characterized by these two parameters. Assuming RTN follows a Markov process, to a first approximation, its kinetics can be described by mean capture and emission times, $\tau_{c}$ and $\tau_{\mathrm{e}}$ respectively.

Several methods to extract these RTN parameters from single devices exist [6-8], but a complete statistically significant description of the RTN requires a large sample size, which makes the characterization a tedious and time-consuming task. Elaborate arrays of transistors have been proposed to facilitate the characterization: arrays of selectable transistors with analog memory [9], with on-chip ADCs [10] and ring oscillators [11]. Comparison of the designs is in Table 1. Our method, verified by measurements on a $28 \mathrm{~nm}$ CMOS test chip (Fig. 1), offers a time-saving solution to both measurement and analysis by full silicon integration.

The method: While $\eta$ and $\mathrm{N}_{\mathrm{T}}$ can be obtained from an array of transistors in an efficient way using the 2-point MSM technique [12], the state-of-the art measurement for extraction of the time constants is the TDDS (Time-Dependent Defect Spectroscopy) [6], which relies on multiple stress and relax phases and a significant data post processing effort. Since the time constants are exponentially distributed with the pdf

$$
f(t)=1 / \tau \cdot e^{-t / \tau}
$$

where both the mean and the spread are defined by $\tau$, we propose to measure the mean value of $\tau_{\mathrm{e}}$ and $\tau_{\mathrm{c}}$ by calculating the average time a trap is charged or neutral in a given time period. In the case of a single active trap, this calculation gives a very good estimate (Fig. 2). In case of multiple traps, an equivalent behavior is obtained (Fig. 3), which provides valid information for modeling the bias temperature instability (BTI) and RTN using the Markov-chain-based model as described in [7].

Hardware implementation: The core of the design consists of an array of 18,144 single nMOS transistors in four different geometries $\left(\mathrm{LW}=[30 \times 100,30 \times 200,30 \times 300,90 \times 100] \mathrm{nm}^{2}\right)($ Fig. 4), similar to [13]. Due to additional transmission gates, these transistors can be measured directly by external equipment or on-chip using the proposed method, depicted in Fig. 5. For the latter, the transistor bias is set by a current DAC (IDAC) and an OPAMP in feedback loop with the selected device under test (DUT). The loop converts RTN induced drain current fluctuations into voltage variations at the DUT gate terminal. This voltage signal is then amplified using the readout circuits which act as a single bit comparator. Finally, the dynamic behavior of the digitized RTN is measured by two counters: one working asynchronously and counting the number of events and the other synchronized with the clock signal counting the number of periods the RTN trap was in the charged or neutral state. The ratio of the results from these two counters gives the sought time constants. All blocks, including the array, are doubled to enable parallel operation. More details about each block follow.

The IDAC was designed to provide a current in the range from 230 $\mathrm{nA}$ to $176 \mu \mathrm{A}$ with 13 bits of accuracy. This is enough to sweep a single or multiple DUTs of any flavor in the array from subthreshold to saturation. The biasing loop OPAMP has a two stage Miller topology and provides up to $60 \mathrm{~dB}$ of open loop gain, a unity gain bandwidth above $10 \mathrm{MHz}$ and a noise floor level lower than $1 \mathrm{mV}$ in amplitude. The High-pass Filter (HPF) removes the DC value of the signal with a cutoff frequency below $10 \mathrm{~Hz}$, thus preserving slower RTN signals. The implementation of the filter is passive and uses diode connected nMOSFETs acting as a $T \Omega$ range resistor (Fig. 5) [14]. Compared to the low-pass solution proposed in [15], the HPF provides (i) the possibility to operate with differential signals and (ii) a well-controlled, smaller range input common-mode for the comparator. The first stage of the readout comparator is a fully differential complementary inverterbased self-biased amplifier [16] with tunable offset (Fig. 6) introduced through modulation of the body bias of the input pMOS transistors. The $\mathrm{V}_{\text {th }}$ tuning range is $75 \mathrm{mV}$ in total (Fig. 7) with a step of $0.22 \mathrm{mV}$. This offset tuning is essential for the method to calibrate the comparator and be able to distinguish RTN events from other noise. The amplifier is constructed with thick oxide transistors to minimize the gate leakage, thus not affecting the load-sensitive HPF. Its differential gain of $30 \mathrm{~dB}$ is enough to relax the input referred noise constraint in the following blocks significantly. The second stage of the comparator is a symmetrical OTA which converts the differential input to a single-ended output. A simple buffer follows to assure the out $B$ (Fig. 5) is rail-to-rail before being digitally processed. Besides the counters, the digital block also includes shift registers to store the data until it is read and frequency dividers for synchronization. It is designed to work for a clock of up to $1 \mathrm{GHz}$, giving an oversampling ratio of 1000 for $1 \mu \mathrm{s}$ long RTN events.

Measurements and results: To assess the time-zero variability, $\mathrm{Id}_{\mathrm{d}}-\mathrm{V}_{g}$ sweeps of all transistors in both arrays have been performed by external SMUs, thus circumventing the on-chip biasing and readout circuits. Measurement results (Fig. 8) are as expected, confirming good operation of the arrays themselves, both measured separately or in parallel.

On-chip measurements of a single RTN trap with $\bar{\tau}_{\mathrm{e}} \sim 0.5 \mathrm{~s}$ have shown that both the biasing and readout circuits work correctly (Fig. 9), proving the design is operational in the frequency range from slightly below $10 \mathrm{~Hz}$. Further testing with external signals supplied at out $A$ (Fig. 5) have proved that the readout block works properly up to $10 \mathrm{MHz}$. The lowest detectable limit of RTN events of $1.2 \mathrm{mV}_{\text {rms }}$ has been measured on RTN-free minimum-size devices in the range $0-20 \mathrm{MHz}$. This value is twice as large compared to simulations, suggesting that the chip is sensitive to environmental noise, which could be reduced by proper shielding. This could be further improved by implementing digital filtering techniques like the median filter.

In Fig. 10, the mean $\bar{\tau}_{\mathrm{e}}$ values extracted from measurements on out $A$ are compared with the values obtained from the counters (out $C$ ). Both results are matching, which proves the method is valid. As commonly observed, in this case the $\bar{\tau}_{\mathrm{e}}$ increases with the increase of the gate bias. Both out $A$ and out $C$ outputs were monitored simultaneously, with the same sampling rate of $1 \mathrm{MS} / \mathrm{s}$ : out $A$ with an oscilloscope and out $C$ with a logic analyzer. In the same time the oscilloscope collected 2000 traces each $10 \mathrm{~ms}$ long equal to $20 \mathrm{MS}$, the logic analyzer collected information equivalent to $1.5 \mathrm{GS}$ in the form of several 64-bit long data-streams. This not only gives a speedup of 75 times, but also a dramatic decrease of output data. Another advantage of the on-chip measurement is that it continues counting new samples while the old are being read out.

Conclusion: A method for on-chip measurement and analysis of dynamic RTN parameters was developed and tested. Test-chip measurements of the design compared to hand measurements showed a significant speedup and a drastic reduction of the data overhead, making the method a valuable RTN measurement and analysis option. 


\begin{tabular}{c|c|c|c|c|c} 
Design & Ref. & Description & Meas. type & Meas. method & Output \\
\hline $\begin{array}{c}\text { Simple } \\
\text { array }\end{array}$ & $\begin{array}{c}{[9]} \\
{[13]}\end{array}$ & $\begin{array}{c}\text { Array of transistors } \\
\text { (with analog memory }{ }^{1} \text { ) }\end{array}$ & $\begin{array}{c}\text { IV curves, RTN } \\
\left(\text { and BTI/HC }{ }^{2} \text { in[13]) }\right.\end{array}$ & External (SMU) & $\begin{array}{c}\text { Raw data } \\
\left(\text { Full time traces }^{3}\right)\end{array}$ \\
\hline $\begin{array}{c}\text { ADC } \\
\text { based }\end{array}$ & {$[10]$} & $\begin{array}{c}\text { On-chip ADC with } \\
\text { preamp./bias circuits }\end{array}$ & IV curves, RTN & On-chip (ADC) & $\begin{array}{c}\text { Raw data } \\
(\text { Full time traces }\end{array}$ \\
\hline $\begin{array}{c}\text { ROSC } \\
\text { based }\end{array}$ & {$[11]$} & $\begin{array}{c}\text { Array of differential } \\
\text { ROSCs }\end{array}$ & $\begin{array}{c}\text { RTN of multiple traps } \\
/ \text { transistors }\end{array}$ & On-chip (counter) & $\begin{array}{c}\text { Digitized RTN traces } \\
\text { (Result of the counters) }\end{array}$ \\
\hline $\begin{array}{c}\text { This } \\
\text { work }\end{array}$ & $\begin{array}{c}\text { Biasing circ., preamp. } \\
\text { and comparator }\end{array}$ & $\begin{array}{c}\tau_{\mathrm{c}} \text { and } \tau_{\mathrm{e}} \text { of single or } \\
\text { multiple traps }\end{array}$ & On-chip (counter) & $\begin{array}{c}\text { Analyzed data } \\
\text { (Result of the counters) }\end{array}$
\end{tabular}

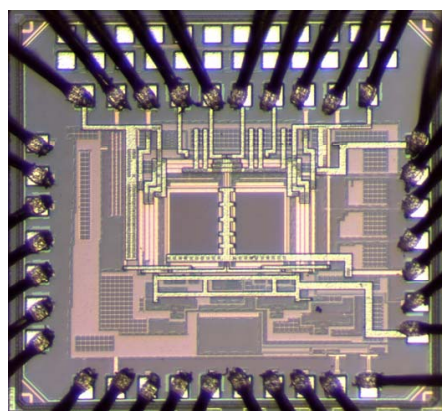

Fig. 1. Bonded chip - microphotograph.

${ }^{1}$ Specific to [9]. ${ }^{2}$ Hot Carrier (HC) stress conditions are limited by the IR drop. ${ }^{3}$ The number of samples is defined by the memory buffer size of the measurement instrument of choice.

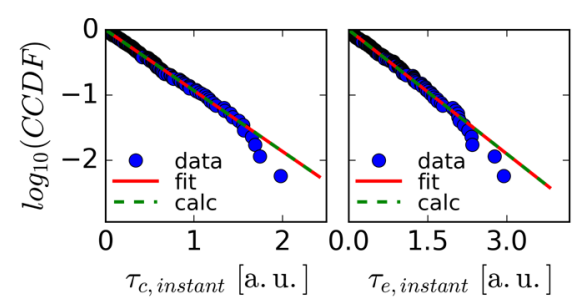

Fig. 2. For a single trap the time-constants are well approximated by the sample mean.

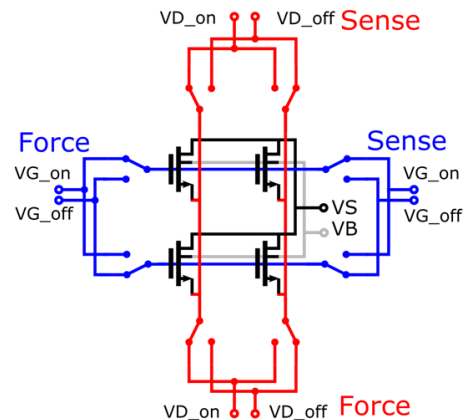

Fig. 4. Simplified representation of the array. The design is equivalent to [13] and includes thick oxide transmission gates, Kelvin sensing on both drain and gate terminals, and 'off' voltages to reduce the leakage.

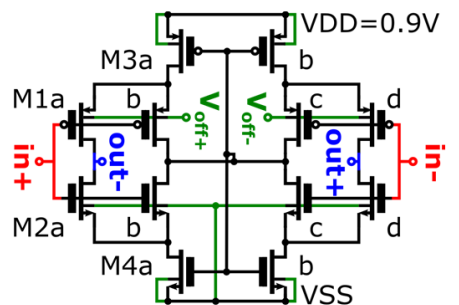

Fig. 6. Schematic of the fully-differential complementary inverter-based self-biased amplifier [16]. The offset compensation is achieved through body bias modulation.
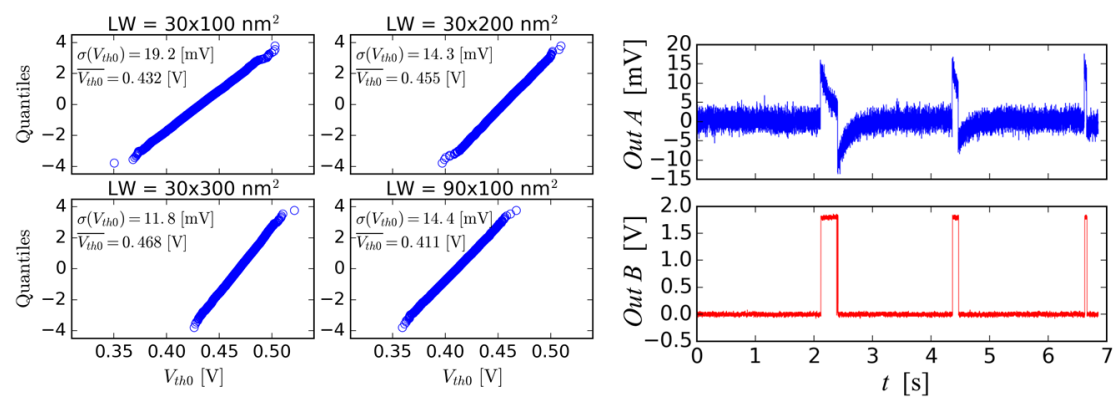

Fig. 8. Time-zero variability measurements reveal Fig. 9. RTN signal from out $A$ (top) and out normal distributions of the $\mathrm{V}_{\text {th } 0}$, with the spread $B$ (bottom). The relative timing is preserved. scaling according to the Pelgrom law [16].

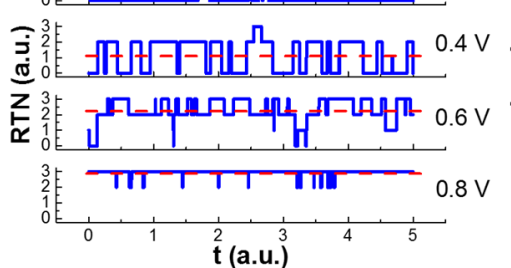

a) ${ }_{2}^{3}$ trap 1 mean $\operatorname{trap} 2 \mathrm{~V}_{\mathrm{gs}}=0.2 \mathrm{~V}$
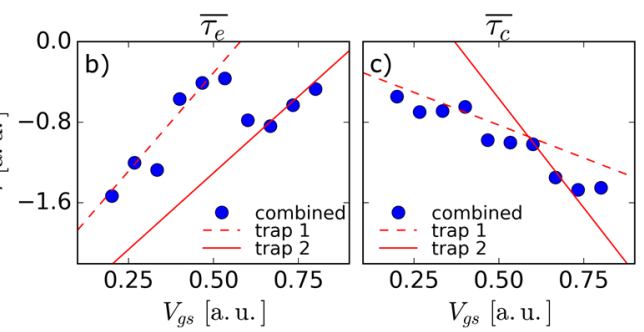

Fig. 3. In the case of multiple traps, depending on their activation energies: 1) an equivalent trap is measured, or 2) traps are observed indirectly through a $\mathrm{V}_{\mathrm{gs}}$ sweep. An example RTN signal from two traps is given in a). In b) and c) the lines represent individual traps, while the dots represent the combined effect.
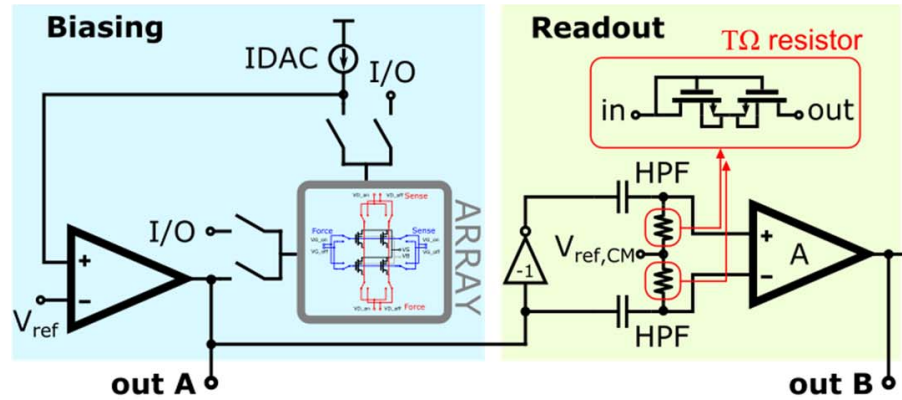

Digital processing

Fig 5. The full circuit implementation of the presented method. The IDAC and the OPAMP in the biasing block set the exact gate voltage needed to make the DUT drain voltage equal to $\mathrm{V}_{\text {ref. }}$ If present, RTN appears at out $A$. The single transistor amplifier ('-1'), HPF and comparator in the readout block amplify the RTN into a digital signal (out B), which is then fed to the counters and read from out $C$.
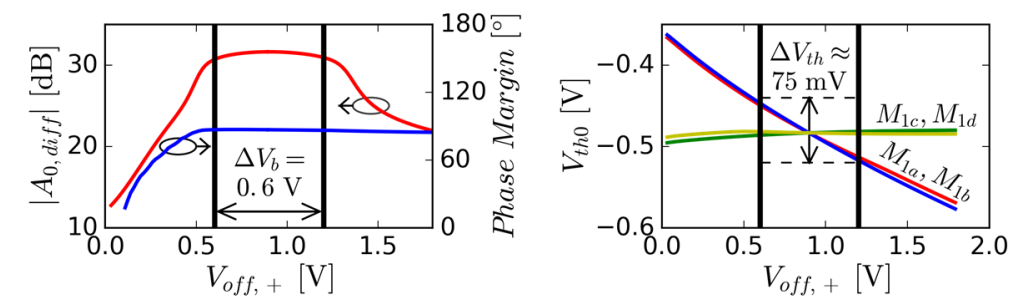

Fig. 7. Body bias modulation in the differential amplifier from Fig. 6 linearly changes the input pMOS threshold voltage and thus the input offset. Within the marked range a $V_{\text {th,offset }}$ of $75 \mathrm{mV}$ is achieved with no significant distortion of the amplifier transfer characteristic.
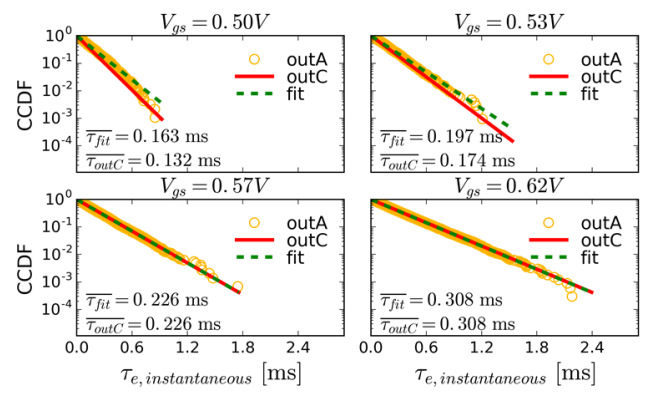

Fig. 10. The circles are RTN events measured at out $A$ and serve as the reference. The dashed green line is a fit. Finally, the full red line is the $\tau_{\mathrm{e}}$ value read from out $C$.

[1] M. Luo et al., TED 62.6, 2015. [2] P. Weckx et al., TED 61.3, 2014. [3] C. Leyris et al., ESSCIRC 2006. [4] A. Asenov et al., TED 50.9, 2003. [5] B. Kaczer et al., IEDL 31.5, 2010. [6] T. Grasser et al., IRPS 2010 [7] P. Weckx et al., IRPS 2015. [8] M. Toledano-Luque et al., IEDM 2013. [9] K. Abe et al., VLSIT 2007. [10] A. Whitcombe et al., ESSDERC 2016. [11] Q. Tang et al., VLSIT 2013. [12] M. Simicic et al., IRPS 2016. [13] M. Simicic et al., IIRW 2015. [14] C. F.

Pereira et al., SBMicro 2016. [15] L.S. Chang et al., IEDL 2013. [16] V. Milovanovic et al., Eurocon 2013. 